\title{
Cocaine Modulation of Frontostriatal Expression of Zif268, D2, and 5-HT2c Receptors in High and Low Impulsive Rats
}

\begin{abstract}
Morgane Besson 1,2,7, Yann Pelloux ${ }^{3}$, Ruth Dilleen ${ }^{1,2}$, David EH Theobald 1,2, Alan Lyon 1,2, Aude Belin-Rauscent ${ }^{4,5}$, Trevor W Robbins ${ }^{1,2}$, Jeffrey W Dalley ${ }^{1,2,6}$, Barry J Everitt ${ }^{*, 1,2,5,8}$ and David Belin ${ }^{4,5,8}$

'Behavioral and Clinical Neuroscience Institute, University of Cambridge, Cambridge, UK; ${ }^{2}$ Department of Psychology, University of Cambridge, Cambridge, UK; Institut de la Timone, CMRS UMR 7289, Marseille, France; ${ }^{4}$ INSERM UI 084, Laboratoire de Neurosciences Expérimentales et Cliniques (LNEC), Université de Poitiers, Team 'Psychobiology of Compulsive Disorders'; Pôle Biologie Santé, Poitiers, France; ${ }^{5}$ INSERM European Associated Laboratory Psychobiology of Compulsive Habits, University of Cambridge/INSERM, University of Poitiers, Poitiers, France; ${ }^{6}$ Department of Psychiatry, University of Cambridge, Addenbrooke's Hospital, Cambridge, UK
\end{abstract}

Impulsivity shares high comorbidity with substance abuse in humans, and high impulsivity $(\mathrm{HI})$ in rats has been identified as a predictive factor for cocaine addiction-like behavior. Despite the evidence that high impulsivity is associated with altered function of corticostriatal networks, the specific neural substrates underlying the increased vulnerability of impulsive individuals to develop cocaine addiction remain unknown. We therefore investigated specific neural correlates of $\mathrm{HI}$ within the corticostriatal circuitry and determined how they interact with a protracted history of cocaine self-administration. We used in situ hybridization to map brain expression of two major genes implicated in impulsivity, encoding the dopamine D2 receptor (DA D2R) and the 5-HT2c receptor (5-HT2cR), and an immediate early gene associated with neuronal plasticity, zif268, in groups of rats selected for $\mathrm{HI}$ and low impulsivity (LI) on a 5-choice serial reaction time task (5-CSRTT) immediately after 5-CSRTT training, and following 10 or 50 days of cocaine self-administration. HI rats exhibited decreased DA D2R mRNA in the mesolimbic pathway, and increased 5-HT2cR mRNA in the orbitofrontal cortex compared with LI rats. $\mathrm{HI}$ rats also showed decreased zif268 mRNA in the ventral and dorsomedial striatum. Cocaine exposure decreased striatal D2R mRNA in both $\mathrm{HI}$ and $\mathrm{LI}$ rats, decreased 5-HT2cR mRNA differentially in striatal and prefrontal areas between $\mathrm{HI}$ and $\mathrm{LI}$ rats, and selectively decreased zif268 mRNA in the orbitofrontal and infralimbic cortices of $\mathrm{HI}$ animals. These findings implicate novel markers underlying the vulnerability of impulsive rats to cocaine addiction that localize to the OFC, infralimbic cortex, and striatum. Neuropsychopharmacology (2013) 38, 1963-1973; doi:I0.1038/npp.2013.95; published online 19 June 2013

Keywords: cocaine; corticostriatal network; gene expression; impulsivity; monoamines; zif268

\section{INTRODUCTION}

High impulsivity is an important feature of a number of psychiatric disorders including drug addiction (VerdejoGarcia et al, 2008). Although the psychological and neurobiological underpinnings of impulsivity are not yet fully understood, accumulating evidence suggests that impulsivity is a major characteristic predisposing individuals to addiction (Verdejo-Garcia et al, 2008). Thus, ADHD patients and individuals with response inhibition deficits display increased vulnerability to develop addiction to nicotine, alcohol, and cocaine (Lee et al, 2011). Consistent with this evidence, high impulsive rats (HI) on the 5-choice serial reaction time task (5-CSRTT), a rodent

\footnotetext{
*Correspondence: Professor BJ Everitt, Department of Psychology, University of Cambridge, Downing Street, Cambridge CB2 3EB, UK. Tel: +44 (0)I2 2333 3583, Fax: +44 (0)I2 23333564 ,

E-mail: bjel0@cam.ac.uk

${ }^{7}$ Current address: Neurobiologie Intégrative des Systèmes Cholinergiques, Institut Pasteur, Paris, France

${ }^{8}$ The last two authors contributed equally to this work.

Received 21 November 2012; revised 14 February 2013; accepted 6

March 2013; accepted article preview online I May 2013
}

analog of the continuous performance task in humans (Robbins 2002), show an increased motivation to selfadminister nicotine (Diergaarde et al, 2008) and higher propensity to escalate cocaine self-administration (Dalley et al, 2007) than low impulsive rats. HI rats also display a greater vulnerability both to develop compulsive cocaine self-administration (Belin et al, 2008) and relapse to cocaine seeking after abstinence (Economidou et al, 2008).

The transition from controlled to uncontrolled, compulsive, cocaine seeking and taking occurs only after a prolonged history of access to the drug (Belin et al, 2009; Deroche-Gamonet et al, 2004; Pelloux et al, 2007; Vanderschuren and Everitt, 2004) and HI rats display a greater propensity than low impulsive rats to undergo the transition to compulsive cocaine self-administration (Belin et al, 2008).

However, even though high impulsivity is known to be associated with altered corticostriatal function, the neurobiological mechanisms governing the emergence of compulsive drug seeking and taking in HI rats are poorly understood. Candidate mechanisms include neurobiological adaptations within frontostriatal circuitry, especially involving DA D2R and 5-HT2cR. Thus, not only have DA D2R 
been reported to be downregulated in both drug addicts (Volkow et al, 2004) and animals following chronic exposure to addictive drugs (Nader et al, 2006), but their availability is also reduced in the ventral but not dorsal striatum of HI rats before any drug exposure (Dalley et al, 2007). 5-HT2cRs are highly expressed in the frontal cortex and the striatum (Clemett et al, 2000) where they are involved in modulating DA neurotransmission (Berg et al, 2008). 5-HT2cRs are also linked to impulsivity (Dalley and Roiser, 2012; Robinson et al, 2008; Xu et al, 2009) and influence the neurochemical and behavioral effects of drugs of abuse, notably by modulating the effects of cocaine and other psychostimulants within mesocorticolimbic circuitry (Bubar et al, 2008; Higgins and Fletcher, 2003). Thus, both D2R and 5-HT2cR are jointly implicated in impulse control and addiction; however, little is known about the way in which these receptors interact with cocaine history in high and low impulsive individuals to modulate neural plasticity and thereby the transition to compulsive cocaine intake (Belin et al, 2008).

In this study, we therefore investigated DA D2R and 5 -HT2cR gene expression in rats identified as HI or LI in the 5-CSRTT both before and following short- or long-term cocaine self-administration. On the basis of our previous work (Deroche-Gamonet et al, 2004; Belin et al, 2008), we hypothesized that neuroadaptive changes occurring after prolonged cocaine self-administration would be most relevant to the emergence of compulsive behavior in $\mathrm{HI}$ rats (Belin et al, 2008). We measured DA D2R and 5 -HT2cR mRNA levels using in situ hybridization in subregions of the prefrontal cortex, as well as in the striatum and its main source of dopaminergic afferents, the ventral tegmental area (VTA) and substantia nigra pars compacta $(\mathrm{SNc})$.

In addition, the long-lasting neurobiological effects of cocaine include the activation of zif268 (Valjent et al, 2006) under the regulation of DA and serotonin (5-HT) transmission (Bath and Baraban, 1993), and are associated with altered synaptic plasticity (Knapska and Kaczmarek, 2004). We therefore also investigated whether the duration of cocaine self-administration differentially recruited this marker of neural plasticity within the corticostriatal networks (Hearing et al, 2008; Unal et al, 2009) of HI and LI rats.

\section{MATERIALS AND METHODS}

\section{Animals}

One hundred and forty-four male Lister hooded rats (Charles River, Kent, UK), weighing approximately $300 \mathrm{~g}$ and about 7 weeks old at the start of the experiments were maintained at $85 \%$ of their free feeding weight during 5-CSRTT training, and housed 4 per cage under humidityand temperature-controlled conditions and an alternating light/dark cycle (red lights on from 07:30 to 19:30 hours; white lights on from 19:30 to $07: 30$ hours). After surgery and during the self-administration experiments, rats were housed individually and fed $20 \mathrm{~g}$ per day, a quantity sufficient to maintain their growth. Water was provided ad libitum. All experimental procedures were conducted in accordance to the UK Animals (Scientific procedures) Act of 1986.

\section{5-CSRTT Training}

Three groups of 48 rats were trained in operant 5-CSRTT chambers controlled by the WhiskerServer software (version 2.8) and FiveChoice client (version 2.6). Each daily session consisted of 100 discrete trials with stable performance being achieved after about 40 sessions. Animals were trained to enter a food magazine to initiate a trial. After an intertrial interval (ITI) of $5 \mathrm{~s}$ had elapsed, a brief light stimulus $(0.5 \mathrm{~s})$ was pseudorandomly presented in one of five apertures. Following a nose-poke in this aperture ('correct' response), animals were rewarded with the delivery of one food pellet ( $45 \mathrm{mg}$ Noyes dustless pellets) in the magazine. A nose-poke response in any of the adjacent apertures ('incorrect response'), or a failure to respond within $5 \mathrm{~s}$ after the onset of the stimulus ('omission'), resulted in no food delivery and a time-out period with the house light extinguished for $5 \mathrm{~s}$. Nose-pokes made during the ITI, that is, before the onset of the stimulus (or 'premature responses') were recorded as a measure of impulsivity, and resulted in a $5 \mathrm{~s}$ time-out and no food reward.

\section{Screening for High and Low Impulsivity}

Following acquisition of the 5-CSRTT, rats were challenged with three long (7s) ITI sessions presented at weekly intervals to encourage impulsive responding, as described previously (Belin et al, 2008; Besson et al, 2010; Dalley et al, 2007; Economidou et al, 2008). Subjects were ranked according to their level of impulsivity throughout the 3 -week screening procedure and the highest and lowest seven subjects selected as HI and LI rats, respectively.

One group of $\mathrm{HI}$ and LI rats was used for in situ hybridization (drug-naive group), whereas the other two groups of HI and LI rats were trained to self-administer cocaine (see below) before subsequent in situ hybridization experiments (Supplementary Figure S1 and Supplementary Table 1).

\section{Surgery}

Rats were deeply anesthetized with ketamine (Ketazet, $90 \mathrm{mg} / \mathrm{kg}$, intraperitoneally; Fort Dodge Animal Health Ltd, Southampton, UK) and xylazine (Rompun, $6.7 \mathrm{mg} / \mathrm{kg}$, intraperitoneally; Bayer, Wuppertal, Germany), and implanted with a catheter (Brian Fromant, Cowley Road, Cambridge, UK) in the right jugular vein (Belin and Everitt, 2008). Rats were given 7 days to recover from surgery before the start of the behavioral experiment.

\section{Self-Administration}

Animals were placed for $2 \mathrm{~h}$ per day in operant chambers different from those used for 5-CSRTT training and in which they were allowed to self-administer a set dose of cocaine $(250 \mu \mathrm{g} /$ injection $)$ under a fixed-ratio 1 schedule of reinforcement. The maximum number of infusions was set to 50 to prevent any differential cocaine intake between the experimental groups. One group of HI and LI rats selfadministered cocaine daily for 11 sessions (short-term cocaine group), whereas another group of HI and LI rats 
self-administered cocaine daily for 44 sessions (long-term cocaine group) (Supplementary Figure S2). These cocaine access periods were based on our earlier research showing that compulsive cocaine taking emerges after atleast 40 daily sessions of cocaine self-administration (Belin et al, 2009, 2011; Deroche-Gamonet et al, 2004; Pelloux et al, 2007; Vanderschuren and Everitt, 2004) specifically in HI rats (Belin et al, 2008). We were interested in the interactions between endophenotypes of impulsivity and the duration of cocaine exposure that subserve the transition to, and not the expression of, compulsivity in $\mathrm{HI}$ rats. We therefore did not probe addiction-like behaviors (Belin et $a l, 2008$ ) in self-administering rats to avoid the differential behavioral responses of $\mathrm{HI}$ and LI rats in the test associated with the measure of compulsivity (resistance to punishment) that could have uncontrolled effects on the molecular markers we studied here.

\section{In Situ Hybridization}

Animals from the short- and long-term cocaine groups were decapitated $60 \mathrm{~min}$ after the last cocaine self-administration session, whereas animals from the drug-naive group were killed after the end of the screening for high and low impulsivity. The in situ hybridization procedure was performed on brain sections with validated oligonucleotide probes for the DA D2R, the 5-HT2cR, and zif268 (Belin et al, 2007). Sections were then exposed at room temperature to Kodak Biomax MR films for different times. Images were captured and analyzed using an image analyzer system for densitometry (Interfocus Imaging, MCID Digital Densitometry System).

\section{Statistical Analysis}

Baseline differences in mRNA levels between HI and LI rats were analyzed with Student's $t$-tests and repeated-measures ANOVAs with group (HI vs LI) as between-subject factor and brain area as within-subject factor (performed separately for the striatum, cortex, and midbrain). For the HI and LI phenotypes, and for each brain area or clusters of brain areas, the comparison between mRNA levels in drugnaive, short- and long-term cocaine rats were analyzed using one-way ANOVAs, with drug treatment as the between-subject factor. Repeated-measures ANOVAs were used to compare mRNA levels in clusters of brain areas between HI and LI rats. All significant main effects $(p<0.05)$ were further analyzed using Student-NewmanKeuls post hoc test. Dimensional inter-relationships were investigated using Pearson's parametric correlation analyses. See detailed methods in Supplementary Information.

\section{RESULTS}

\section{In Situ Hybridization}

A highly specific and homogeneous macroscopic signal was obtained with each probe for every region of interest (Supplementary Figures S3-5). All the optical densities were within the range of linearity of the X-ray films.

\section{Short- and Long-Term Cocaine Self-Administration in $\mathrm{HI}$ and LI Rats}

In both self-administration experiments, that is, short(Supplementary Figure S2a) and long- (Supplementary Figure S2b) term cocaine exposure, HI rats differed from LI rats neither in their acquisition of instrumental responding for cocaine nor in their total amount of cocaine intake per session. By the end of the self-administration sessions, rats had been exposed to a cumulative dose of self-infused cocaine of $109 \pm 8$ and $98 \pm 4 \mathrm{mg}$ in short-term $\mathrm{HI}$ and LI groups, respectively (Supplementary Figure S2c), and of $462 \pm 20$ and $475 \pm 34 \mathrm{mg}$ in long-term HI and LI groups, respectively (Supplementary Figure S2d).

\section{Basal Differences in mRNA Levels Between HI and LI Rats}

HI rats exhibited higher zif268 mRNA levels than LI rats in the nucleus accumbens shell (NAcbS) and core (NAcbC) $(t=2.249, \quad p=0.048, \quad \mathrm{df}=10$ and $t=2.460, \quad p=0.034$, $\mathrm{df}=10$, respectively) and in the dorsomedial striatum (DMS) $(t=4.046, p=0.0023, \mathrm{df}=10) \quad$ (Figure 1a). The highly significant effect observed in the DMS was confirmed by repeated-measure ANOVAs (group effect: $\mathrm{F}(1,10)=8.12$, $p<0.05$; brain area effect $\mathrm{F}(3,30)=54.07, p \leqslant 0.001)$ and subsequent post hoc analysis $(p=0.005)$. This differential profile of zif268 mRNA levels within the striatum between drug-naive $\mathrm{HI}$ and LI rats was accompanied by alterations in DA D2R (Figure 1b) and 5-HT2cR (Figure 1c) mRNA levels (Figure 5). Compared with LI rats, HI rats showed lower DA D2R mRNA levels in the NAcbS $(t=-2.313$, $p=0.043, \mathrm{df}=10)$ and the VTA $(t=-2.600, p=0.026$, $\mathrm{df}=10$ ) but higher 5 -HT2cR mRNA levels in the orbitofrontal cortex (OFC) $(t=2.235, p=0.049, \mathrm{df}=10)$, which was confirmed by repeated-measure ANOVAs (brain area effect: $F(5,50)=50,30, p \leqslant 0.001$; group $\times$ brain area interaction: $F(5,50)=4.95, p \leqslant 0.001)$, and subsequent post hoc analysis $(p=0.000187)$. There was a significant correlation between DA D2R mRNA levels in the VTA and the SNc in LI rats $(R=0.618, p \leqslant 0.05)$, whereas this was not the case in $\mathrm{HI}$ rats $(R=0.361, \mathrm{NS})$. 5-HTR2cR mRNA levels in the DMS and dorsolateral striatum (DLS) were correlated in HI rats, as they were between the different regions of the prefrontal cortex, except the OFC, which was not the case for LI animals, where 5-HT2cR mRNA levels were correlated between the NAcbS and NAcbC (Supplementary Table 2).

\section{Effect of Short- and Long-Term Cocaine Self-Administration on zif268 mRNA Levels in HI and LI Rats}

Differential effects were observed on zif268 mRNA levels in $\mathrm{HI}$ and LI rats after cocaine self-administration (Figure 2). Three-way repeated-measures ANOVAs performed for striatal areas showed a group $\times$ drug treatment interaction $\left(\mathrm{F}_{2,28}=4.18, p<0.05\right)$ with a main effect of brain area $\left(\mathrm{F}_{3,84}=98.23, p \leqslant 0.001\right)$, brain area $\times$ drug treatment interaction $\left(\mathrm{F}_{6,84}=4.89, p<0.001\right)$, and brain area $\times$ group $\times$ drug treatment interaction $\left(\mathrm{F}_{6,84}=2.22, p<0.05\right)$. Newman-Keuls post hoc tests showed significant differences between drug-naive and short-term cocaine LI rats in the DMS $(p=0.0014)$. For cortical areas, three-way 

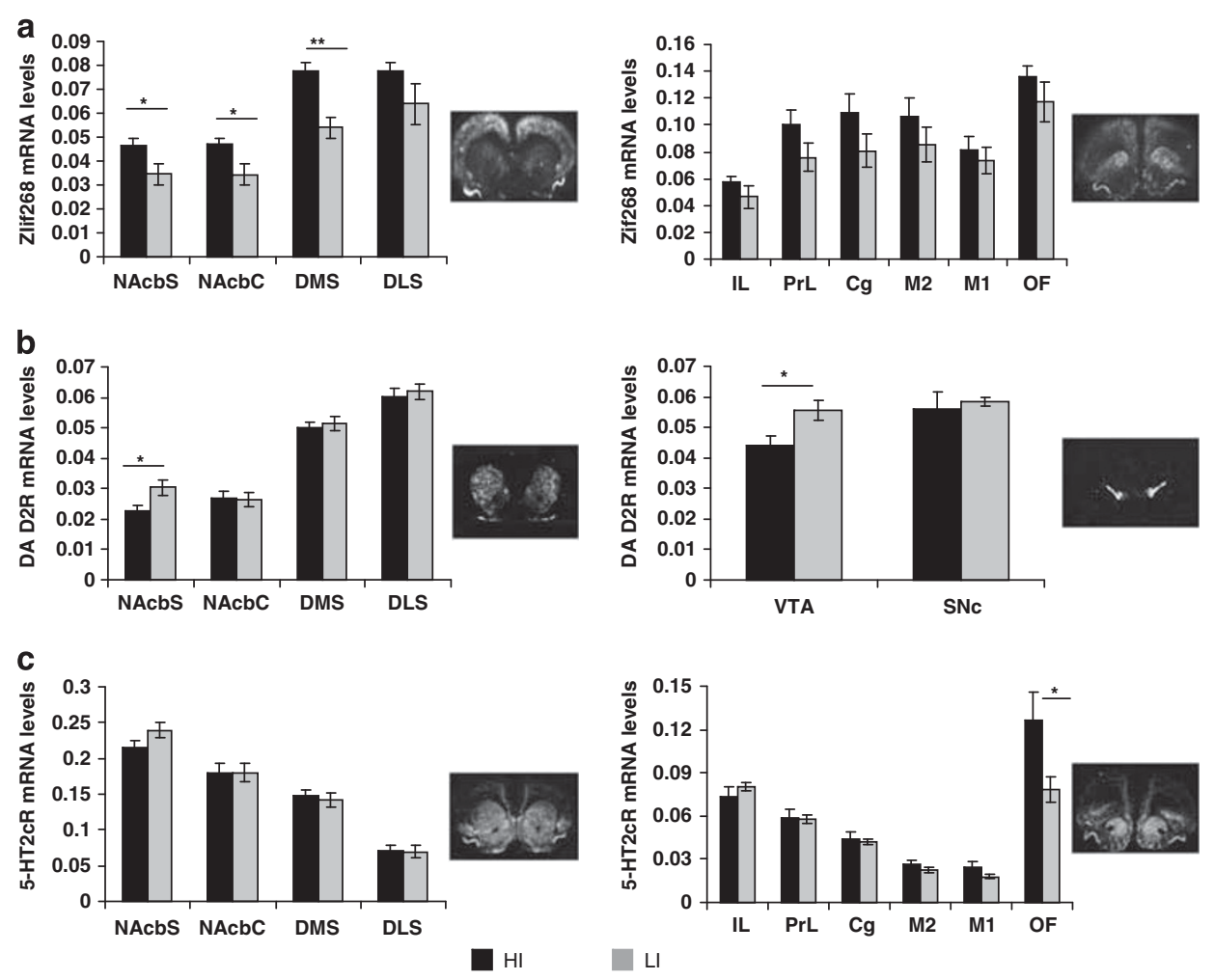

Figure I Differences in mRNA levels (expressed as mean \pm SEM) between high impulsive $(H \mathrm{I})$ (black bars) and low impulsive (LI) (gray bars) rats for (a) zif268 (HI, $n=6$; LI, $n=6$ ), (b) dopamine D2 receptor (DA D2R) (HI, n=5; LI, $n=7)$, and (c) 5-HT2c receptor $(5-H T 2 c R)(H I, n=6 ; L I, n=6)$. The optical densities were measured in the nucleus accumbens shell (NAcbS), nucleus accumbens core (NAcbC), dorsomedial striatum (DMS), dorsolateral striatum (DLS) (for zif268, DA D2R, and 5-HT2cR); the ventral tegmental area (VTA) and substantia nigra pars compacta (SNc) (for DA D2R); and the infralimbic (IL), prelimbic (PrL), cingulate (Cg), secondary motor (M2), primary motor (MI), and orbitofrontal (OF) cortices (for zif268 and 5-HT2cR) of HI and LI drug-naive animals. $* p \leqslant 0.05, * * * \leqslant 0.01$.

ANOVAs showed a significant main effect of brain area $\left(\mathrm{F}_{5,150}=104.39, \quad p \leqslant 0.001\right)$ and brain area $\times$ drug treatment interaction $\left(\mathrm{F}_{10,150}=1.98, p<0.05\right)$. Upon confirmation of these main effects, analyses were then completed with ANOVAs performed separately in $\mathrm{HI}$ and LI groups.

In $\mathrm{HI}$ rats but not in LI rats, zif268 mRNA levels were decreased after cocaine self-administration in the cortex (drug exposure: $\mathrm{F}_{2,15}=4.203, p<0.05$; cortical structure: $\left.\mathrm{F}_{2,5}=66.392, p<0.001\right)$. This effect was observed after both short- $(p=0.017)$ and long-term cocaine exposure $(p=0.014)$. ANOVA restricted to each cortical area showed that this effect was limited to the infralimbic (IL) cortex $\left(\mathrm{F}_{2,15}=4.98, p<0.05\right)$ and $\mathrm{OFC}\left(\mathrm{F}_{2,15}=5.66, p<0.05\right)$ of $\mathrm{HI}$ rats, regardless of the duration of cocaine exposure. Post hoc analyses confirmed that HI rats exposed to short- and longterm cocaine self-administration displayed decreased zif268 mRNA levels in the IL ( $p=0.037$ and 0.016 , respectively) and the OFC ( $p=0.018$ and 0.019 , respectively).

By contrast, cocaine produced different effects on zif268 mRNA levels in the striatum of LI rats (drug exposure: $\mathrm{F}_{2,14}=4.15, \quad p<0.05$; drug exposure $\times$ striatal structure interaction: $\mathrm{F}_{2,6}=6.15, p<0.001$ ) (Figure $2 \mathrm{~b}$ ). Thus in LI rats, an increase in zif268 mRNA levels was observed after cocaine self-administration in the DMS $\left(\mathrm{F}_{2,15}=12.94\right.$, $p<0.001)$ after short- $(p=0.0001)$ but not long-term $(p=0.2521)$ cocaine exposure. No quantitative differences in zif268 mRNA levels were observed in the IL and OFC between drug-naive and cocaine-exposed LI rats.

These differential changes in zif268 mRNA levels between HI and LI rats were further emphasized by repeatedmeasures ANOVAs showing that zif268 mRNA levels were significantly decreased following cocaine self-administration in the IL and OFC of $\mathrm{HI}$ rats compared with LI rats (group effect: $F_{1,22}=4.84, p<0.05$ ) (Figure 5).

\section{Effect of Short- and Long-Term Cocaine Self-Administration on DA D2R mRNA Levels in $\mathrm{HI}$ and LI Rats}

$\mathrm{HI}$ and LI rats displayed different neurobiological adaptions to the duration of cocaine self-administration with regard to D2 mRNA levels. Three-way repeated-measures ANOVAs for striatal areas revealed a group $\times$ drug treatment interaction $\left(\mathrm{F}_{2,28}=10.62, \quad p<0.01\right)$, a brain area effect $\left(\mathrm{F}_{3,84}=372.73, p \leqslant 0.001\right)$, a brain area $\times$ drug treatment interaction $\left(\mathrm{F}_{6,84}=4.18, p<0.001\right)$, as well as a brain area $\times$ group $\times$ drug treatment $\left(\mathrm{F}_{6,84}=2.38, p=0.0357\right)$. NewmanKeuls post hoc tests showed significant differences between drug-naive and short-term cocaine LI rats in the DMS $(p=0.002)$ and DLS $(p=0.0002)$. For midbrain areas (VTA and SNc), global ANOVAs showed a group effect $\left(\mathrm{F}_{1,32}=6.95, p<0.05\right)$ and a brain area effect $\left(\mathrm{F}_{1,32}=8.09\right.$, 

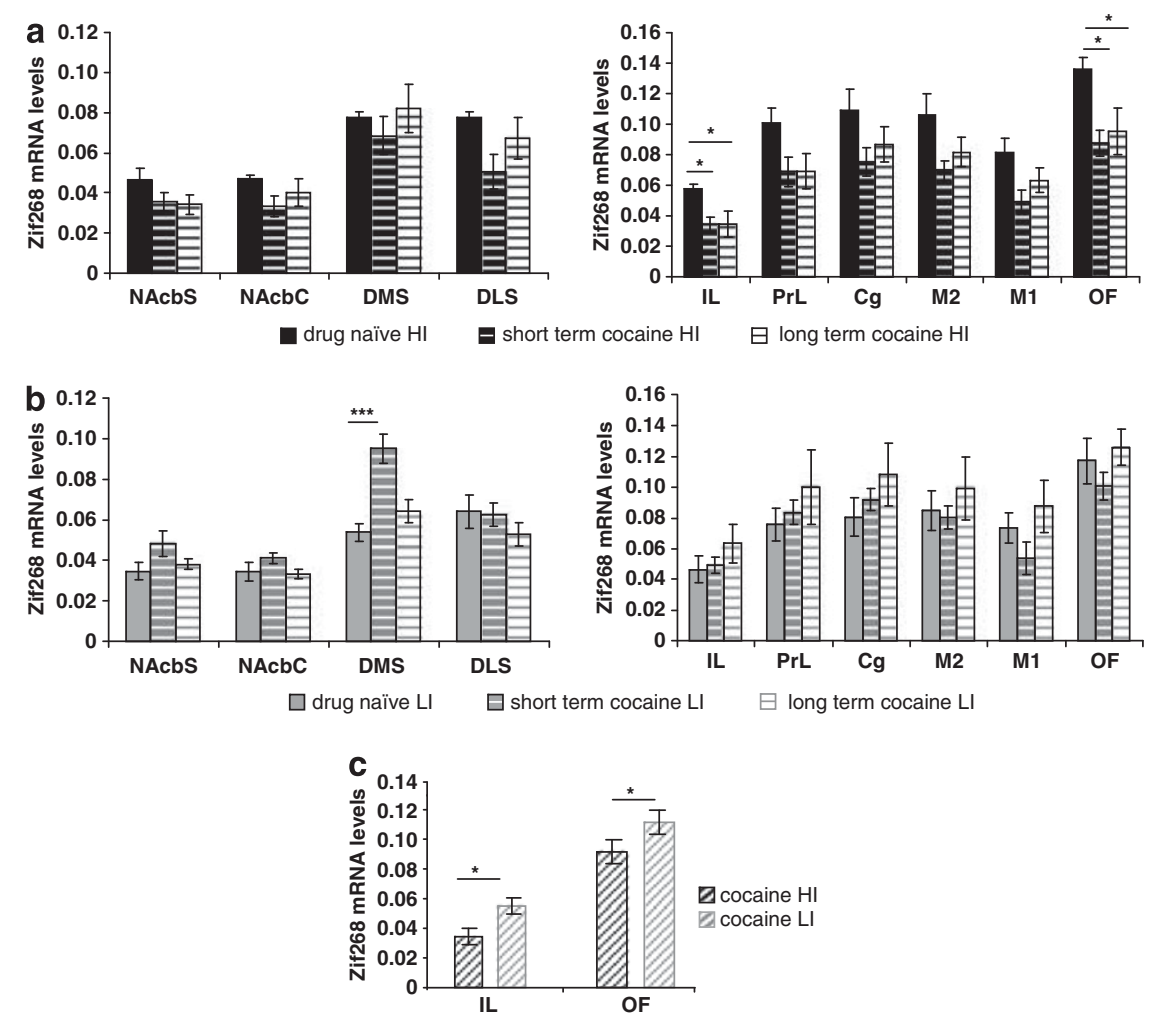

Figure 2 Effects of short- and long-term cocaine self-administration on zif268 mRNA levels (expressed as mean \pm SEM) in high impulsive (HI) and low impulsive (LI) rats. (a) Zif268 mRNA levels in drug-naive (black plain bars, $n=6$ ), short-term cocaine (black bars with white stripes, $n=6$ ), and long-term cocaine (white bars with black stripes, $n=7$ ) HI rats; (b) zif268 mRNA in drug-naive (gray plain bars, $n=6$ ), short-term cocaine (gray bars with white stripes, $n=7$ ), and long-term cocaine (white bars with gray stripes, $n=5$ ) LI rats; (c) zif268 mRNA in pooled short- and long-term cocaine HI (black bars with white diagonals, $n=13$ ) and LI (gray bars with gray diagonals, $n=12$ ) rats. The optical densities were measured in the nucleus accumbens shell (NAcbS), nucleus accumbens core (NAcbC), dorsomedial striatum (DMS), dorsolateral striatum (DLS), and in the infralimbic (IL), prelimbic (PrL), cingulate (Cg), secondary motor (M2), primary motor (MI), and orbitofrontal (OF) cortices $* p \leqslant 0.05$, **** $p \leqslant 0.001$.
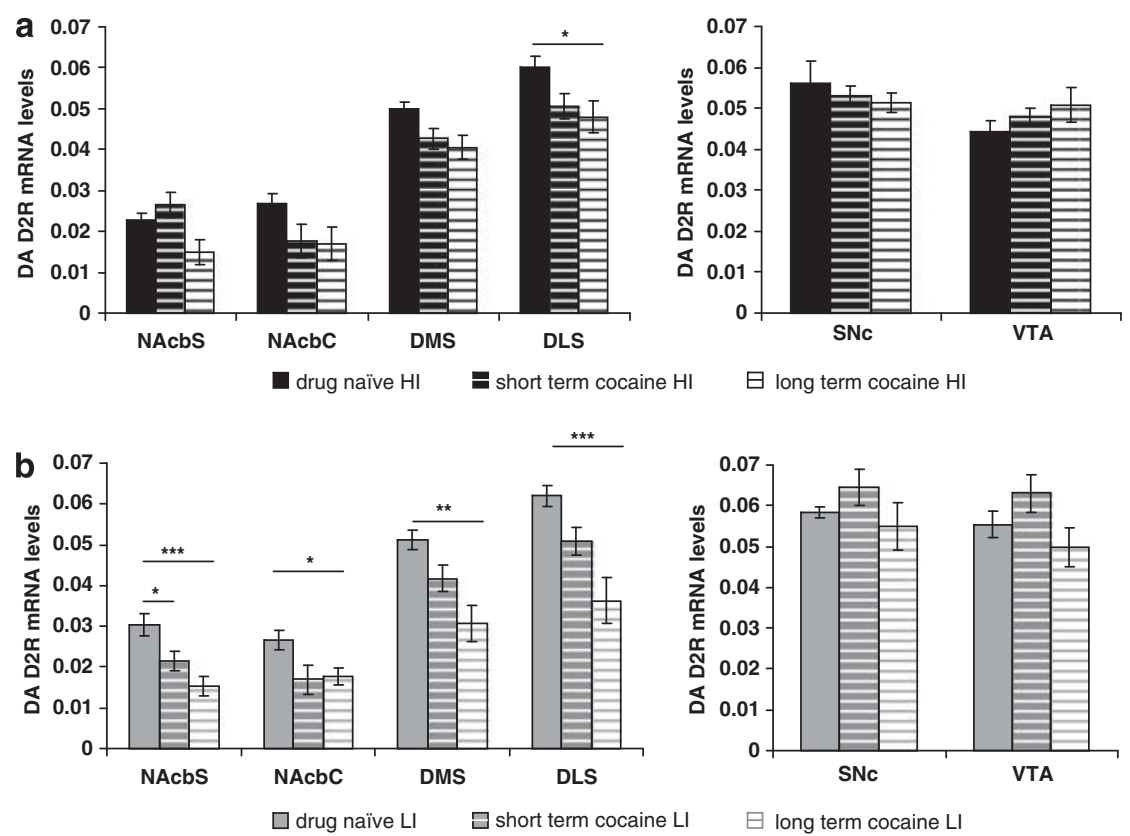

Figure 3 Effects of short- and long-term cocaine self-administration on dopamine D2 receptor (DA D2R) mRNA levels (expressed as mean \pm SEM) in high impulsive (HI) and low impulsive (LI) rats; (a) DA D2R mRNA levels in drug-naive (black plain bars, $n=5$ ), short-term cocaine (black bars with white stripes, $n=5$ ), and long-term cocaine (white bars with black stripes, $n=6) H \mathrm{Hats}$; (b) DA D2R mRNA levels in drug-naive (gray plain bars, $n=7$ ), shortterm cocaine (gray bars with white stripes, $n=7$ ), and long-term cocaine (white bars with gray stripes, $n=5$ ) LI rats. The optical densities were measured in the nucleus accumbens shell (NAcbS), nucleus accumbens core (NAcbC), dorsomedial striatum (DMS), dorsolateral striatum (DLS), ventral tegmental area (VTA), and substantia nigra pars compacta (SNc). $* p \leqslant 0.05, * * p \leqslant 0.01, * * * p \leqslant 0.001$. 
$p<0.01)$. Analyses were then completed with ANOVAs performed separately in HI and LI groups.

In HI rats, cocaine self-administration resulted in decreased DA D2R mRNA levels in the striatum (drug $\times$ structure interaction: $F_{2,6}=3.537, p<0.01$ ) (Figure $3 \mathrm{a}$ ), an effect mainly attributed to the DLS $\left(\mathrm{F}_{2,12}=3.45, p<0.05\right)$ and the $\operatorname{NAcbS}\left(\mathrm{F}_{2,13}=4.60, p<0.05\right)$, where DA D2R were lower in $\mathrm{HI}$ rats exposed to long-term cocaine self-administration than in drug-naive HI rats $(p=0.045$ and 0.074 , respectively) (Figure 5).

In LI rats, cocaine self-administration was associated with decreased DA D2R mRNA levels in the dorsal and ventral striatum (drug $\times$ structure interaction: $F_{2,6}=3.83$, $p<0.05$ ) (Figure $3 \mathrm{~b}$ ). This effect was significant in each striatal structure, namely the NAcbS $\left(\mathrm{F}_{2,17}=9.44, p<0.01\right)$, $\operatorname{NAcbC}\left(\mathrm{F}_{2,17}=3.74, p<0.05\right)$, DMS $\left(\mathrm{F}_{2,17}=8.93, p<0.01\right)$, and DLS $\left(\mathrm{F}_{2,17}=11.06, p<0.01\right)$. Post hoc analyses revealed that this effect was significant after both short- $(n=7)$ and long-term $(n=5)$ cocaine self-administration in the $\operatorname{NAcbS}(p=0.019$ and 0.001 , respectively), and after longterm cocaine self-administration in the NAcbC $(p=0.039)$, the DMS $(p=0.002)$, and the DLS $(p=0.0006)$ (Figure 5).
Effect of Short- and Long-Term Cocaine Self-Administration on 5-HT2cR mRNA Levels in HI and LI Rats

The history of cocaine self-administration differentially altered 5-HT2cR mRNA levels in the corticostriatal circuitry of HI and LI rats. Three-way repeated-measures ANOVAs performed for striatal areas showed an effect of drug treatment $\left(\mathrm{F}_{2,30}=4.97, p<0.05\right)$ and brain area $\left(\mathrm{F}_{3,90}=393.90, p \leqslant 0.001\right)$. For cortical areas, global ANOVAs showed a brain area effect $\left(\mathrm{F}_{5,150}=0.13, p \leqslant 0.001\right)$ and a brain area $\times$ group $\times$ drug treatment $\left(F_{10,150}=2.75\right.$, $p<0.01)$. Analyses were then completed with ANOVAs performed separately in HI and LI groups.

5-HT2cR mRNA levels in HI rats were significantly decreased after cocaine self-administration in the cingulate $(\mathrm{Cg})$ cortex and the NAcbS $\left(\mathrm{F}_{2,16}=3.95, \quad p<0.05\right.$; $\mathrm{F}_{2,15}=4.95, p<0.05$, respectively) (Figure 4a). Post hoc analyses revealed that this decrease was observed after long( $p=0.019)$ but not short-term $(p=0.307)$ cocaine exposure in the NAcbS, opposite to that observed in the Cg cortex ( $p=0.007$ and $p=0.060$ for short- and long-term cocaine self-administration, respectively) (Figure 5).
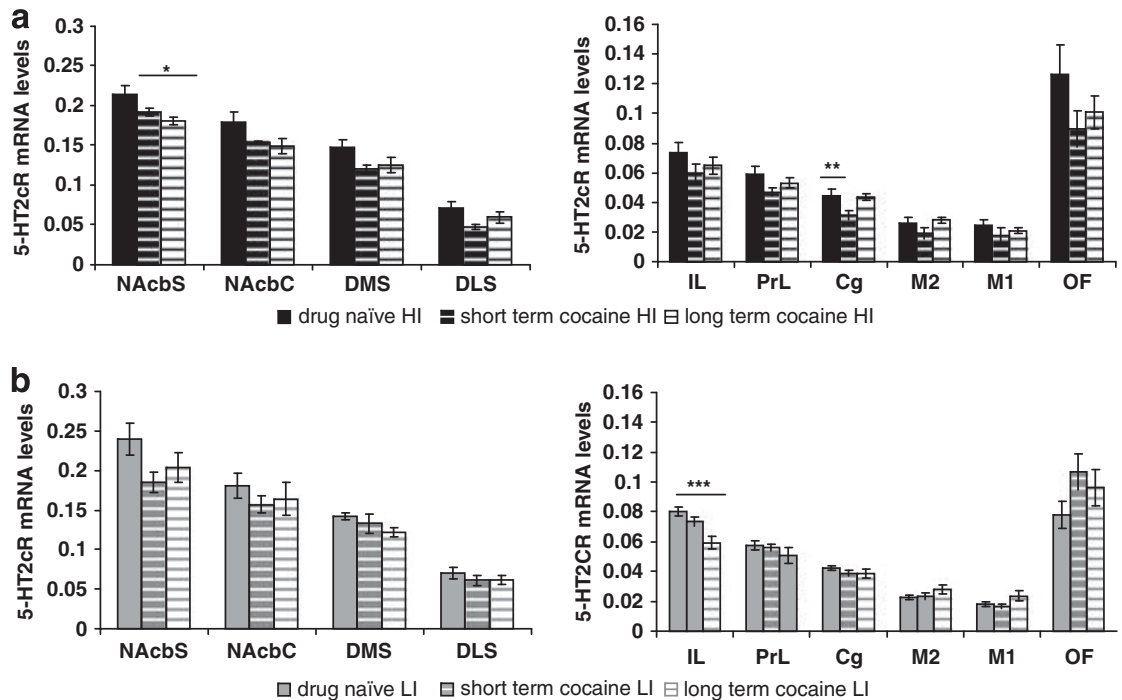

Figure 4 Effects of short- and long-term cocaine self-administration on 5-HT2c receptor (5-HT2cR) mRNA levels (expressed as mean \pm SEM) in high impulsive $(\mathrm{HI})$ and low impulsive (LI) rats. (a) $5-\mathrm{HT} 2 \mathrm{cR}$ mRNA levels in drug-naive (black plain bars, $n=6$ ), short-term cocaine (black bars with white stripes, $n=6$ ), and long-term cocaine (white bars with black stripes, $n=6$ ) HI rats; (b) 5-HT2cR mRNA in drug-naïve (gray plain bars, $n=6$ ), short-term cocaine (gray bars with white stripes, $n=7$ ), and long-term cocaine (white bars with gray stripes, $n=5) \mathrm{LI}$ rats. The optical densities were measured in the nucleus accumbens shell (NAcbS), nucleus accumbens core (NAcbC), dorsomedial striatum (DMS), dorsolateral striatum (DLS), and in the infralimbic (IL), prelimbic (PrL), cingulate $(\mathrm{Cg})$, secondary motor $(\mathrm{M} 2)$, primary motor $(\mathrm{MI})$, and orbitofrontal (OF) cortices. * $p \leqslant 0.05$, *** $\leqslant 0.0 \mathrm{I}$, ***** $p \leqslant 0.001$.

Figure 5 Schematic of the main differences observed between high impulsive $(\mathrm{HI})$ and low impulsive (LI) rats in the course of cocaine self-administration history. $\mathrm{HI}$ in rats was associated with a differential profile of expression, within the corticostriatal circuitry, of two important monoamine receptors involved the regulation of impulse control and drug reinforcement, and of the plasticity-associated gene, zif268. Compared with $\mathrm{LI}$ rats, drug-naive HI rats displayed increased 5-HT2c receptor (5-HT2cR) mRNA levels in the orbitofrontal cortex (OFC) and lower D2R mRNA levels in the ventral tegmental area (VTA) and NAcbS. HI rats had a higher level of zif268 gene expression in the nucleus accumbens shell (NAcbS), nucleus accumbens core (NAcbC), and dorsomedial striatum (DMS) compared with LI rats. Cocaine self-administration resulted in different neuroadaptations within the corticostriatal circuitry of HI and $\mathrm{LI}$ rats. Cocaine exposure was associated with a decrease in 5-HT2cR mRNA levels, specifically in the anterior cingulate (Cg) cortex and the NAcbS of $\mathrm{HI}$ rats after short- and long-term self-administration, respectively, and in the infralimbic (IL) cortex of LI rats after long-term self-administration. This was paralleled by a decrease in dopamine D2 receptor (DA D2R) mRNA levels in the striatum of both $\mathrm{HI}$ and $\mathrm{LI}$ rats. This effect, which encompassed the entire striatum of LI rats, was more evident after long-term cocaine self-administration and mostly involved the DLS in HI rats. Zif268 mRNA levels were decreased after short- and long-term cocaine self-administration in the IL and OF cortices of HI rats specifically. By contrast, in LI rats, zif268 mRNA levels were increased in the DMS after short-term cocaine self-administration. 
By contrast, cocaine self-administration resulted in a decrease in 5-HT2cR mRNA levels in the IL cortex of LI rats $\left(\mathrm{F}_{2,15}=8.77, p<0.05\right)$, after long- $(p=0.001)$ but not short-term $(p=0.176)$ exposure to the drug (Figures $2 \mathrm{~b}$ and 5).

Correlational analyses further showed that inter-regional patterns of 5-HT2cR expression were also differentially reorganized after cocaine exposure in $\mathrm{HI}$ and LI rats (see Supplementary Table 2).

In summary, as compared with LI animals, HI rats were characterized by decreased DA D2R mRNA levels in the NAcb shell and the VTA, as well as increased 5-HT2cR mRNAs level in the OFC. HI rats also displayed higher level of zif268 gene expression in the NAcbS, NAcbC, and DMS.

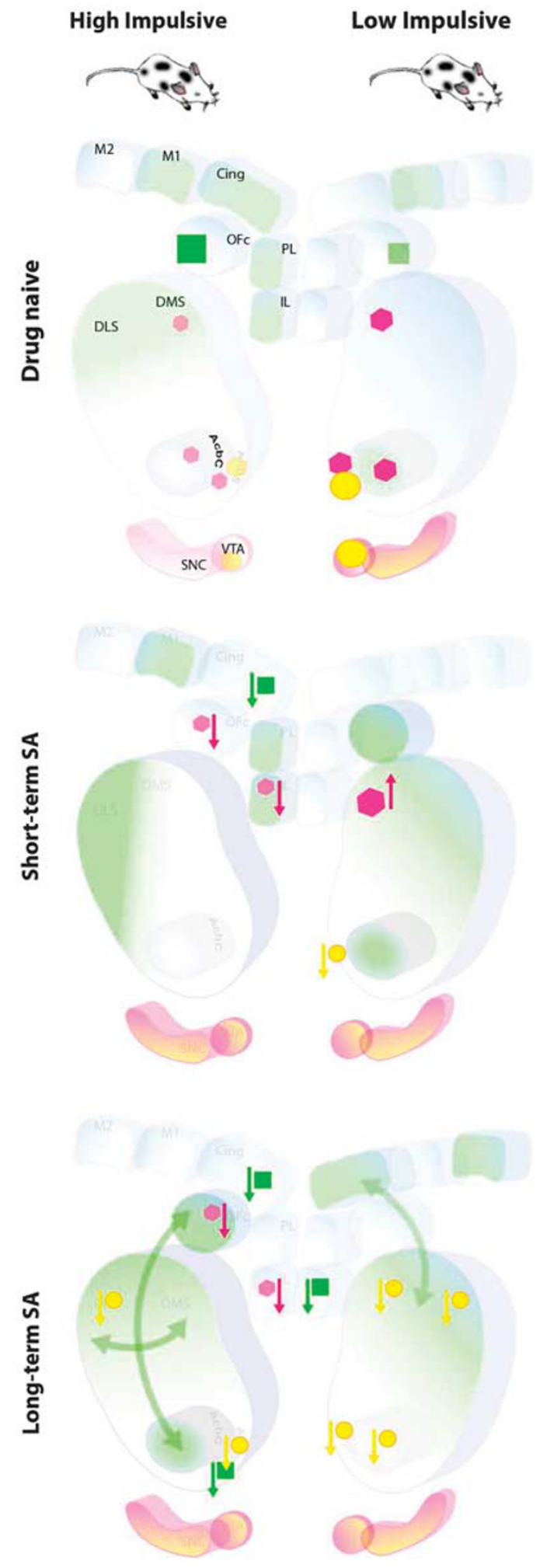


Zif268 mRNA levels were decreased after short- and longterm cocaine self-administration in the IL and OF cortices of HI rats, whereas in LI rats, zif268 mRNA levels were increased in the DMS after short-term cocaine.

DA D2R mRNA levels were decreased after cocaine exposure in the striatum of both HI and LI rats. This effect encompassed the entire striatum of LI rats, was more evident after long-term cocaine self-administration, and mostly involved the DLS in HI rats.

Cocaine exposure was also associated with a decrease in 5-HT2cR mRNA levels in the anterior Cg cortex and the NAcbS of HI rats after short- and long-term selfadministration, respectively, and in the IL cortex of LI rats after long-term self-administration.

The main results are summarized in Figure 5.

\section{DISCUSSION}

Increasing evidence suggests that impulse control deficits, as observed in highly impulsive subjects, may contribute to increased vulnerability to develop addiction (VerdejoGarcia et al, 2008). Accordingly, high impulsivity on the 5-CSRTT has been shown to predict several hallmarks of cocaine addiction (Dalley et al, 2007; Belin et al, 2008). HI rats have been shown to be more vulnerable than LI rats to escalate cocaine self-administration when given extended and intermittent access to cocaine (Dalley et al, 2007) and to switch from controlled to compulsive cocaine seeking, that persists despite aversive foot-shocks, and which emerges after at least 40 days of cocaine selfadministration (Belin et al, 2008). Furthermore, HI rats are more vulnerable to relapse after protracted abstinence (Economidou et al, 2008). However, HI did not differ from LI rats in their propensity to acquire cocaine selfadministration (this study; Dalley et al, 2007; Belin et al, 2008; Economidou et al, 2008) nor in their average baseline intake during the $2 \mathrm{~h}$ daily self-administration sessions. Thus, the transition to compulsive drug seeking observed in HI rats after an extended cocaine self-administration history stems from the interaction between the drug and neurobiological substrates that are specific to those subjects (Everitt et al, 2008; Dalley et al, 2011). In this study, we used this lack of differential cocaine intake between HI and LI rats to identify neurobiological correlates of protracted cocaine self-administration that may contribute to the transition to compulsivity in vulnerable subjects. In doing so, we did not directly assess addiction-like criteria in HI and LI rats because this would have resulted in uncontrolled differential behaviors and differing quantities of cocaine intake between the two groups.

Previous studies have established that highly impulsive subjects have lower levels of DA D2/D3 receptor availability in the midbrain and ventral striatum but not in the nigrostriatal system (Buckholtz et al, 2010; Dalley et al, 2007; Lee et al, 2009). These findings, demonstrating that $\mathrm{HI}$ and LI rats display similar DA D2R mRNA levels in the nigrostriatal system but major differences in the mesolimbic system not only support these observations, but further specify the neurobiological locus of decreased DA D2R in the striatal system associated with the HI trait, namely the shell, but not the core, of the NAcb, and also the VTA. HI on the 5-CSRTT has been associated in vitro with increased DA release in the NAcbS (Diergaarde et al, 2008), whereas lower midbrain DA D2/3R availability in highly impulsive humans has also been proposed to be associated with enhanced DA activity (Buckholtz et al, 2010). DA D2R are expressed on GABA neurons within the ventral striatum, and also on VTA DA neurons where they are presynaptic and function as autoreceptors that regulate DA release associated with behavioral activation (Viggiano et al, 2003).

It is well documented that individuals addicted to cocaine and other drugs have lower DA D2R availability in the striatum when studied during abstinence (Volkow et al, 2004). Moreover, in monkeys with a history of cocaine self-administration, there is also a marked reduction in DA D2 receptors in the ventral striatum under short access conditions, which persists, and also progresses to the dorsal striatum after chronic self-administration (Porrino et al, 2004). In accord with these observations, we observed reduced DA D2R mRNA levels in the NAcb and the dorsal striatum of rats after cocaine self-administration, with a larger decrease in dorsal striatal areas after long-term selfadministration. These reductions were found in both $\mathrm{HI}$ and LI rats, thereby suggesting that cocaine exposure interacts with other neurobiological vulnerability markers in impulsive individuals.

A considerable body of evidence links the 5-HT system with impulsivity (Dalley and Roiser, 2012), with a significant role for 5-HT2cRs (Dalley and Roiser, 2012; Robinson et al, 2008; Xu et al, 2009). Here we observed higher levels of 5-HT2cR mRNA selectively in the OFC of HI rats. The OFC has an important role in response inhibitory control (Everitt et al, 2007) and damage to this area increases impulsivity in rodents (Eagle and Baunez, 2010). OFC 5-HT depletion decreases behavioral flexibility in monkeys (Clarke et al, 2007), which has been proposed to result from 5-HT2cR supersensitivity (Boulougouris and Robbins, 2010). Conversely, 5-HT2cR antagonism within the OFC improves cognitive flexibility in rats (Boulougouris and Robbins, 2010).

We further observed that 5-HTR2cR mRNA levels in the DMS and DLS were correlated in HI rats, as they were between different regions of the medial prefrontal cortex. This apparent convergent $5-\mathrm{HT} 2 \mathrm{cR}$ regulation throughout the cortex and dorsal striatum in HI rats was in contrast to the lack of correlation between DA D2R mRNA levels in the VTA and SNc of these animals, which was instead apparent in LI rats. There is evidence that alteration in 5-HT activity influences impulsive behavior through a modulation of DA transmission (Harrison et al, 1997; Oades, 2008).

In this study, 5-HT2cR mRNA levels were decreased in the NAcbS of HI rats after long-term cocaine selfadministration. Although previous studies have highlighted the role of 5-HT2cRs in psychostimulant action (Bubar and Cunningham, 2008), the consequences of drug exposure on these receptors has not been established to date (Filip et al, 2010). 5-HT2cRs control mesocorticolimbic DA activity (Di Matteo et al, 2002) and 5-HT2cRs expressed in the NAcbS modulate NAcb DA outflow (Navailles et al, 2006). Drug-induced modifications in 5-HT2cR gene expression may contribute to the changes in striatal DA activity that have been proposed to underlie the shift from impulsivity to 
compulsive drug seeking occurring during the development of addiction (Everitt et al, 2008).

HI rats displayed a different profile of expression of the plasticity-related immediate-early gene zif268 mRNA, the levels of which were increased in the ventral and dorsomedial striatum compared with LI subjects. This is in marked contrast to the differential response to cocaine in $\mathrm{HI}$ and LI rats in that, HI but not LI rats displayed a decrease in zif268 mRNA levels selectively in the IL and OF cortices after short-term cocaine self-administration. This decrease persisted even after protracted exposure to the drug, suggesting altered function of these frontal areas after exposure to addictive drugs in vulnerable individuals.

There is a close link between zif268 gene expression and plasticity-linked neuronal activity (Knapska and Kaczmarek, 2004) and here we investigated zif268 gene expression as a potential marker of plasticity engaged by DA and 5-HT transmission. ZIF268 protein acts as a transcription factor controlling the expression of downstream genes implicated in the regulation of neuronal responses to various stimuli. High levels of zif268 mRNA in the ventral and dorsal striatum of $\mathrm{HI}$ animals suggest greater synaptic plasticity occurring in these neural loci after 5-CSRTT training only in animals with weak inhibitory control. However, the decrease in zif268 mRNA levels in the IL and OFC of HI rats after short- and long-term cocaine self-administration, as compared with 5-CSRTT training, suggests a cocaine-induced loss of neuronal plasticity in these brain areas. Persistent OFC dysfunction has been demonstrated in humans addicted to cocaine and other drugs, as well as in rats even after relatively brief exposure to cocaine (Ersche et al, 2011; Schoenebaum and Shaham, 2008; Everitt et al, 2007). Dysfunction of this area leads to the emergence of inflexible and compulsive behavior, including compulsive drug seeking, a hallmark feature of drug addiction that is predicted by high impulsivity on the 5-CSRTT (Belin et al, 2008). In accordance with our present findings showing alterations in both OFC zif268 and NAcbS 5-HT2cR gene expression in HI after cocaine self-administration, it has been shown that OFC lesions both induce compulsive behavior in rats and cause a decrease in the content of striatal 5-HT (Schilman et al, 2010).

The IL cortex plays a role in the expression and consolidation of extinction of drug seeking (Millan et al, 2011) and inactivation of this area causes the reinstatement of previously extinguished drug seeking, whereas activation of the IL prevents reinstatement (Peters et al, 2008). The present data showing decreased zif268 mRNA levels in this area after cocaine taking in HI rats are in accord with the increased susceptibility to relapse to cocaine seeking in these rats (Economidou et al, 2008). Decreased neuronal plasticity in the OFC and IL after cocaine self-administration may therefore also provide a mechanism for the emergence of compulsive drug taking in high impulsive individuals when exposed to specific conditions.

Zif268 gene expression has been shown to be increased in the ventral and dorsal striatum after acute cocaine administration and to return to pre-cocaine levels or be decreased after long-term cocaine exposure (Fumagalli et al, 2009; Hope et al, 1992). Similarly, we observed increased zif268 mRNA levels in the DMS of LI rats after short- but not long-term cocaine self-administration. By contrast, in HI rats, no changes in zif $268 \mathrm{mRNA}$ levels were observed in striatal areas after cocaine taking, suggesting that LI individuals have a greater propensity than $\mathrm{HI}$ individuals to display neuronal plasticity in response to cocaine. This observation is consistent with the recent demonstration that rats vulnerable to addiction-like behavior for cocaine are characterized by a lack of metaplasticity, the so-called anaplasticity, which corresponds to an inability to counteract drug-induced impairments in synaptic plasticity, within the frontostriatal networks (Kasanetz et al, 2010, 2012).

In LI rats, 5-HT2cR mRNA levels were decreased after long access but not after short access to cocaine in the IL cortex. In contrast to $\mathrm{HI}$ animals, no other changes were observed selectively in LI rats after long-term cocaine selfadministration. In LI rats, zif268 mRNA levels were increased in the DMS after short- but not after long-term cocaine, indicating that neuroadaptations occur in the brain of these rats after long-term exposure to the drug. These data suggest that drug exposure has less persistent consequences for brain functioning in low impulsive rats compared with high impulsive rats.

The observed differences between $\mathrm{HI}$ and LI rats in the effects of cocaine self-administration on the DA D2R and 5-HT2cR mRNA levels illustrate at the gene expression level neurobiological adaptations to protracted drug exposure that occur differentially in vulnerable and nonvulnerable subjects, as also described by Kasanetz et al $(2010,2012)$ at the synaptic plasticity level. It also allowed us to rule out a potential confounding effect on gene expression of cocaine self-administered in the last session (but see Belin et al, 2007).

The differential drug-induced alterations in gene expression and plasticity observed in HI and LI rats could not be attributed to a differential effect of aging in these two subpopulations, not only because there is little if any evidence for a baseline shift in the expression of DA D2R and 5-HT2cRs in young adults over a period of 10 weeks, which is the larger window between the first long ITI and the last self-administration session of this study, but also because a potential differential effect of age on subpopulations of rats of the same strain has not been demonstrated previously. The effects observed here could, nevertheless, be attributable to various components of the self-administration history that are not directly related to overall drug exposure, namely instrumental training or protracted exposure to the self-administration context and associated Pavlovian stimuli that have been reported to modify DA D2R and DAT mRNA levels in sensitized animals (Belin et al, 2007).

The lack of assessment of compulsive aspects of addiction in this study, however, does not allow the direct conclusion that the adaptations observed here in HI rats subserve compulsive cocaine intake. Instead, they may reflect the development of a particular neurobiological state preceding the expression of compulsive drug use in vulnerable subjects. Little differential cocaine-induced plasticity on gene expression was observed in HI rats, suggesting that further modifications may be necessary to facilitate the emergence of compulsive cocaine selfadministration. 
In conclusion, we show that $\mathrm{HI}$ in rats is associated with regional differences in the expression of genes related to monoamine neurotransmission and neuronal plasticity in striatal and frontal areas, including decreased mesolimbic DA D2R mRNAs, increased OFC 5-HT2cR mRNAs, and decreased zif268 mRNAs in ventral and dorsal striatal areas. This study therefore suggests that cocaine exposure differentially alters 5-HT2cR and DA D2R mRNAs between $\mathrm{HI}$ and LI rats and that, when exposed to cocaine self-administration, the former display decreased zif268 mRNA levels in the IL and OF cortices, suggesting a dysfunction of these frontal areas after exposure to addictive drugs in vulnerable individuals. This may, in part, underlie the compulsive drug seeking and high rate of relapse observed in HI rats after protracted cocaine seeking (Belin et al, 2008; Economidou et al, 2008). In high impulsive individuals, basal or constitutive alterations in frontostriatal circuitry, especially regarding monoamine neurotransmission, might confer vulnerability to further drug-induced impairments in prefrontal cortical function, eventually favoring the development of cocaine addiction.

\section{ACKNOWLEDGEMENTS}

This study was supported by the MRC (G9536855, G1002231 and G0701500) and by a joint core award from the MRC and Wellcome Trust (MRC G1000183; WT 093875/Z/10/Z) in support of the Behavioural and Clinical Neuroscience Institute at Cambridge University. $\mathrm{MB}$ was supported by an EMBO fellowship. DB and ABR were supported by an INSERM AVENIR Grant, the AXA research fund, the fondation pour la recherche médicale (FRM), and the IREB. AL was supported by a Wellcome Trust Programme Grant (089589/Z/09/Z). We thank Mercedes Arroyo for technical assistance with the in situ hybridization experiments.

\section{DISCLOSURE}

The authors declare no conflict of interest.

\section{REFERENCES}

Bath RV, Baraban JN (1993). Activation of transcription factor genes in striatum by cocaine: role of both serotonin and dopamine systems. J Pharmacol ExpTher 267: 496-505.

Belin D, Balado E, Piazza PV, Deroche-Gamonet V (2009). Pattern of intake and drug craving predict the development of cocaine addiction-like behavior in rats. Biol Psychiatry 65: 863-868.

Belin D, Berson N, Balado E, Piazza PV, Deroche-Gamonet V (2011). High-novelty-preference rats are predisposed to compulsive cocaine self-administration. Neuropsychopharmacology 36: $569-579$.

Belin D, Deroche-Gamonet V, Jaber M (2007). Cocaine-induced sensitization is associated with altered dynamics of transcriptional responses of the dopamine transporter, tyrosine hydroxylase, and dopamine $\mathrm{D} 2$ receptors in $\mathrm{C} 57 \mathrm{Bl} / 6 \mathrm{~J}$ mice. Psychopharmacology (Berl) 193: 567-578.

Belin D, Everitt BJ (2008). Cocaine seeking habits depend upon dopamine-dependent serial connectivity linking the ventral with the dorsal striatum. Neuron 57: 432-441.

Belin D, Mar AC, Dalley JW, Robbins TW, Everitt BJ (2008). High impulsivity predicts the switch to compulsive cocaine-taking. Science 320: 1352-1355.
Berg KA, Clarke WP, Cunningham KA, Spampinato U (2008). Fine-tuning serotonin2c receptor function in the brain: molecular and functional implications. Neuropharmacology 55: 969-976.

Besson M, Belin D, McNamara R, Theobald DE, Castel A, Beckett VL et al (2010). Dissociable control of impulsivity in rats by dopamine $\mathrm{D} 2 / 3$ receptors in the core and shell subregions of the nucleus accumbens. Neuropsychopharmacology 35: 560-569.

Boulougouris V, Robbins TW (2010). Enhancement of spatial reversal learning by 5 -HT2C receptor antagonism is neuroanatomically specific. J Neurosci 30: 930-938.

Bubar MJ, Cunningham KA (2008). Prospects for serotonin 5-HT2R pharmacotherapy in psychostimulant abuse. Prog Brain Res 72: 319-346.

Buckholtz JW, Treadway MT, Cowan RL, Neil D, Woodward ND, $\mathrm{Li} \mathrm{R}$ et al (2010). Dopaminergic network differences in human impulsivity. Science 329: 532.

Clarke HF, Walker SC, Dalley JW, Robbins TW, Roberts AC (2007). Cognitive inflexibility after prefrontal serotonin depletion is behaviorally and neurochemically specific. Cereb Cortex 17: 18-27.

Clemett DA, Punhani T, Duxon MS, Blackburn TP, Fone KC (2000; Immuno-histochemical localisation of the 5-HT2c receptor protein in the rat CNS. Neuropharmacology 39: 123-132.

Dalley JW, Everitt BJ, Robbins TW (2011). Impulsivity, compulsivity, and top-down cognitive control. Neuron 69: 680-694.

Dalley JW, Fryer TD, Brichard L, Robinson ES, Theobald DE, Lääne $\mathrm{K}$ et al (2007). Nucleus accumbens D2/3 receptors predict trait impulsivity and cocaine reinforcement. Science 315: 1267-1270.

Dalley JW, Roiser JP (2012). Dopamine, serotonin and impulsivity. Neuroscience 215: 42-58.

Deroche-Gamonet V, Belin D, Piazza PV (2004). Evidence for addiction-like behavior in the rat. Science 305: 951-953.

Di Matteo V, Cacchio M, Di Giulio C, Esposito E (2002). Role of serotonin(2C) receptors in the control of brain dopaminergic function. Pharmacol Biochem Behav 71: 727-734.

Diergaarde L, Pattij T, Poortvliet I, Hogenboom F, de Vries W, Schoffelmeer AN et al (2008). Impulsive choice and impulsive action predict vulnerability to distinct stages of nicotine seeking in rats.. Biol Psychiatry 63: 301-308.

Eagle DM, Baunez C (2010). Is there an inhibitory-responsecontrol system in the rat? Evidence from anatomical and pharmacological studies of behavioral inhibition. Neurosci Biobehav Rev 34: 50-72.

Economidou D, Pelloux Y, Robbins TW, Dalley JW, Everitt BJ (2008). High impulsivity predicts relapse to cocaine-seeking after punishment-induced abstinence. Biol Psychiatry 65: 851-856.

Ersche KD, Barnes A, Jones PS, Morein-Zamir S, Robbins TW, Bullmore ET (2011). Abnormal structure of frontostriatal brain systems is associated with aspects of impulsivity and compulsivity in cocaine dependence. Brain 134: 2013-2024.

Everitt BJ, Belin D, Economidou D, Pelloux Y, Dalley JW, Robbins TW (2008). Neural mechanisms underlying the vulnerability to develop compulsive drug-seeking habits and addiction. Philos Trans $R$ Soc Ser B 363: 3125-3135.

Everitt BJ, Hutcheson D, Ersche KD, Pelloux Y, Dalley JW, Robbins TW (2007). The orbital prefrontal cortex and drug addiction in laboratory animals and humans. Ann NY Acad Sci 1121: 576-597.

Filip M, Alenina N, Bader M, Przegaliński E (2010). Behavioral evidence for the significance of serotoninergic (5-HT) receptors in cocaine addiction. Addict Biol 15: 227-249.

Fumagalli F, Franchi C, Caffino L, Racagni G, Riva MA, Cervo L (2009). Single session of cocaine intravenous self-administration shapes goal-oriented behaviors and up-regulates Arc mRNA levels in rat medial prefrontal cortex. Int J Neuropsychopharmacol 12: 423-429. 
Harrison AA, Everitt BJ, Robbins TW (1997). Central 5-HT depletion enhances impulsive responding without affecting the accuracy of attentional performance: interactions with dopaminergic mechanisms. Psychopharmacology (Berl) 133: 329-342.

Hearing MC, See RE, McGinty JF (2008). Relapse to cocaineseeking increases activity-regulated gene expression differentially in the striatum and cerebral cortex of rats following short or long periods of abstinence. Brain Struct Funct 213: 215-227.

Higgins GA, Fletcher PJ (2003). Serotonin and drug reward: focus on 5-HT2C receptors. Eur J Pharmacol 480: 151-162.

Hope B, Kosofsky B, Hyman SE, Nestler EJ (1992). Regulation of immediate early gene expression and AP-1 binding in the rat nucleus accumbens by chronic cocaine. Proc Natl Acad Sci USA 89: $5764-5768$.

Kasanetz F, Deroche-Gamonet V, Berson N, Balado E, Lafourcade $\mathrm{M}$, Manzoni $\mathrm{O}$ et al (2010). Transition to addiction is associated with a persistent impairment in synaptic plasticity. Science 328: 1709-1712.

Kasanetz F, Lafourcade M, Deroche-Gamonet V, Revest JM, Berson N, Balado E et al (2012). Prefrontal synaptic markers of cocaine addiction-like behavior in rats. Mol Psychiatry (E-pub ahead of print).

Knapska E, Kaczmarek L (2004). A gene for neuronal plasticity in the mammalian brain: Zif268/Egr-1/NGFI-A/Krox-24/TIS8/ ZENK? Prog Neurobiol 74: 183-211.

Lee B, London ED, Poldrack RA, Farahi J, Nacca A, Monterosso JR et al (2009). Striatal dopamine D2/D3 receptor availability is reduced in methamphetamine dependence and is linked to impulsivity. J Neurosci 29: 14734-14740.

Lee SS, Humphreys KL, Flory K, Liu R, Glass K (2011). Prospective association of childhood attention-deficit/hyperactivity disorder (ADHD) and substance use and abuse/dependence: a metaanalytic review. Clin Psychol Rev 3: 328-341.

Millan EZ, Marchant NJ, McNally GP (2011). Extinction of drug seeking. Behav Brain Res 217: 454-462.

Nader MA, Morgan D, Gage HD, Nader SH, Calhoun TL, Buchheimer $\mathrm{N}$ et al (2006). PET imaging of dopamine D2 receptors during chronic cocaine self-administration in monkeys. Nat Neurosci 9: 1050-1056.

Navailles S, Moison D, Ryczko D, Spampinato U (2006). Regiondependent regulation of mesoaccumbens dopamine neurons in vivo by the constitutive activity of central serotonin2C receptors. J Neurochem 99: 1311-1319.

Oades RD (2008). Dopamine-serotonin interactions in attentiondeficit hyperactivity disorder (ADHD). Prog Brain Res 172: 543-565.

Pelloux Y, Everitt BJ, Dickinson A (2007). Compulsive drug seeking by rats under punishment: effects of drug taking history. Psychopharmacology (Berl) 194: 127-137.
Peters J, LaLumiere RT, Kalivas PW (2008). Infralimbic prefrontal cortex is responsible for inhibiting cocaine seeking in extinguished rats. J Neurosci 28: 6046-6053.

Porrino LJ, Daunais JB, Smith HR, Nader MA. (2004). The expanding effects of cocaine: studies in a nonhuman primate model of cocaine self-administration. Neurosci Biobehav Rev 27: 813-820.

Robbins TW (2002). The 5-choice serial reaction time task: behavioural pharmacology and functional neurochemistry (2002). Psychopharmacology (Berl) 163: 362-380.

Robinson ES, Dalley JW, Theobald DE, Glennon JC, Pezze MA, Murphy ER et al (2008). Opposing roles for 5-HT2A and 5-HT2C receptors in the nucleus accumbens on inhibitory response control in the 5-choice serial reaction time task. Neuropsychopharmacology 33: 2398-2406.

Schilman EA, Klavir O, Winter C, Sohr R, Joel D (2010). The role of the striatum in compulsive behavior in intact and orbitofrontalcortex-lesioned rats: Possible involvement of the serotonergic system. Neuropsychopharmacology 35: 1025-1039.

Schoenbaum G, Shaham Y (2008). The role of orbitofrontal cortex in drug addiction: a review of preclinical studies. Biol Psychiatry 63: 256-262.

Unal CT, Beverley JA, Willuhn I, Steiner H (2009). Long-lasting dysregulation of gene expression in corticostriatal circuits after repeated cocaine treatment in adult rats: effects on zif 268 and homer 1a. Eur J Neuroci 29: 1615-1626.

Valjent E, Aubier B, Corbillé AG, Brami-Cherrier K, Caboche J, Topilko P et al (2006). Plasticity-associated gene Krox24/Zif268 is required for long-lasting behavioral effects of cocaine. J Neurosci 26: 4956-4960.

Vanderschuren LJ, Everitt BJ (2004). Drug seeking becomes compulsive after prolonged cocaine self-administration. Science 305: 1017-1019.

Verdejo-Garcia A, Lawrence JA, Clark L (2008). Impulsivity as a vulnerability marker for substance-use disorders: review of findings from high-risk research, problem gamblers and genetic association studies. Neurosci Biobehav Res 32: 777-810.

Viggiano D, Ruocco LA, Sadile AG (2003). Dopamine phenotype and behaviour in animal models: in relation to attention deficit hyperactivity disorder. Neurosci Biobehav Rev 27: 623-637.

Volkow ND, Fowler JS, Wang GJ, Swanson JM. (2004). Dopamine in drug abuse and addiction: results from imaging studies and treatment implications. Mol Psychiatry 9: 557-569.

Xu X, Brookes K, Sun B, Ilott N, Asherson P (2009). Investigation of the serotonin $2 \mathrm{C}$ receptor gene in attention deficit hyperactivity disorder in UK samples. BMC Res Notes 2: 71.

Supplementary Information accompanies the paper on the Neuropsychopharmacology website (http://www.nature.com/npp) 